\title{
Laboratory equipment for testing of hollow-fibre membrane properties
}

\author{
J. Suchánková ${ }^{1}$, L. Herecová ${ }^{1}$, D. Plachá ${ }^{2}$, P. Danihelka ${ }^{1}$ \\ \& O. Martiník ${ }^{1}$ \\ ${ }^{1}$ Laboratory of Risk Research and Management, Faculty of Safety \\ Engineering, VSB - Technical University of Ostrava, Czech Republic \\ ${ }^{2}$ Nanotechnology Centre, \\ VSB - Technical University of Ostrava, Czech Republic
}

\begin{abstract}
Water has a major role at present. Its usage is determined by its quality, which is supported by regulations in many countries. In real life, technology using membrane elements is widely used for water purification. Commonly, membrane modules in the form of hollow fibres are applied. There are modules made from different porous and polymeric materials on the market. Laboratory equipment was constructed to compare different commercially available membranes in this study. The equipment was designed for the testing of hollow-fibre membranes and for observing various parameters during the performance of volatile organic compound (toluene) separation.

The device allows testing either parameters of individually selected hollowfibre membranes or parallel measurements of the commercially available module as a whole to compare with an individual fibre.

The equipment was used for pilot testing in laboratory conditions. Efficiencies of toluene separation from water under various flow rates by using several commercially available membrane modules were observed. Experiments confirmed decreasing separation capability with increasing water flow rates. Choice of membrane material is very significant for further applications because the results showed that the separation efficiency varies with the use of different materials.
\end{abstract}

Keywords: water treatment, hollow-fibre, volatile, pervaporation. 


\section{Introduction}

Laboratory equipment for testing the selected parameters of commercially available hollow-fibre membranes was designed and constructed (Fig. 1). The equipment is applicable for testing various parameters influencing the separation of volatile compounds from contaminated water. The equipment can also be used for testing hydrophobic and hydrophilic material properties, membrane separation efficiency for selected individual compounds, pressure resistance, chemical resistance and others.

Selected parameters can be quantified by using the equipment and the efficiencies of different membranes can consequently be compared. The module with a single membrane fibre or module with a fibre bundle or both modules in parallel can be used for selected membrane parameter testing. Simultaneously several single-fibre modules can be used in parallel with the equipment.

Two different mediums - liquid and gaseous phases - occur in the equipment during the testing. They come into mutual contact only in the membrane pores of the module. From this perspective, the module can be used in two arrangements: 1) the gas phase passes through the inside of the fibres and water flows outside, 2) the liquid phase passes through the fibre inside and gas passes outside. It may be effective to use both options for initial testing and subsequently determine the most appropriate method for a given situation.

It is possible to set direction and flow rates for the convection of liquid and gas phases by using regulatory elements. Water flow rate is regulated by using a threaded valve that is located in the output from a pump and also in output from a module. Due to this arrangement we can regulate water pressure in a module. The sensitivity of water flow settings is $0.21 / \mathrm{min}$. Air flow inside the modules is set by two-way PTFE valves. The valves are connected in line before and after the module to regulate air flow and pressure inside fibres. It is possible to use needle valves or flow meters with control. The described equipment was designed so that water flow in a module with a fibre bundle is to a range of 0.5 $6 \mathrm{l} / \mathrm{min}$, in a module with single fibre to a range of $50-1000 \mathrm{ml} / \mathrm{min}$.

There are several options of experiment arrangements. Convections in a module can be conducted in a co-current or counter-current flow arrangement of the liquid and gaseous phases. Experiments can be realized in linear or closed (circulate) settings. Testing can be performed in static or dynamic conditions of both phases.

Pilot experiments were conducted on the basis of principle of pervaporation when a volatile organic compound is evaporated from water to a gas medium. The volatile organic compound was removed by gaseous medium (air) outside of a module. Toluene was selected as the volatile compound in experiments that were aimed at testing separation efficiency (i) in different water flow rates in a separation unit (module) and (ii) with using different commercially available membranes. 


\section{Experimental}

\subsection{Equipment description}

The laboratory equipment consists of a large-capacity storage tank, two modules (one with single fibre and the second with a fibre bundle) and pump, valves, hosepipe, flow-meters (see Figure 1).

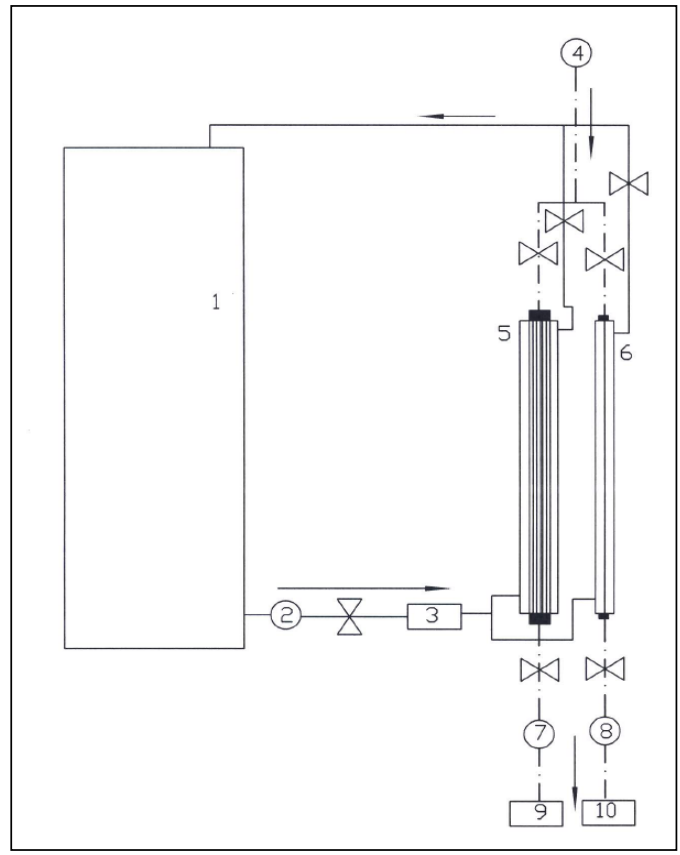

Figure 1: Experimental arrangement for the evaluation of membrane properties.

Water contaminated by a defined quantity of toluene was stored in the largecapacity tank (1), which was connected to the modules with hosepipes from inert polymer material (5 and 6). Water is pumped through the equipment with a pump (2), continuous flow control (0-9 1/min) is performed with a valve located in the line after the pump. Water volume in the tank in respect of water flow through modules and experiment duration is sufficient so an important decrease of toluene concentration in water does not occur.

The water circuit is divided in two parts to supply each module with contaminated water directly from tank. After flowing through the modules the water is returned back to the tank; it is cyclic water circuit. The flow-meter for flow control is located after the pump (3).

A compressed air source (compressor) is the air source for modules and it is located before the air input to the modules (4). The air phase on the module 
output can be released on the outside of the equipment (not recommended) or it can pass through an adsorbent, such as activated carbon adsorption tubes ( 7 and 8). They can be used consequently for analysis. The air flow can be control with a flow-meter added to circuit line (9 and 10).

Single-fibre modules were prepared for the characterization process on single fibre (Figure 2). The fibre (1) is stretched through plastic T-joint (2) and glass tube (3). The plastic joint is attached to the tube by using a silicon seal. Hosepipes for air and water intake are attached to the relevant T-joints ends (5).

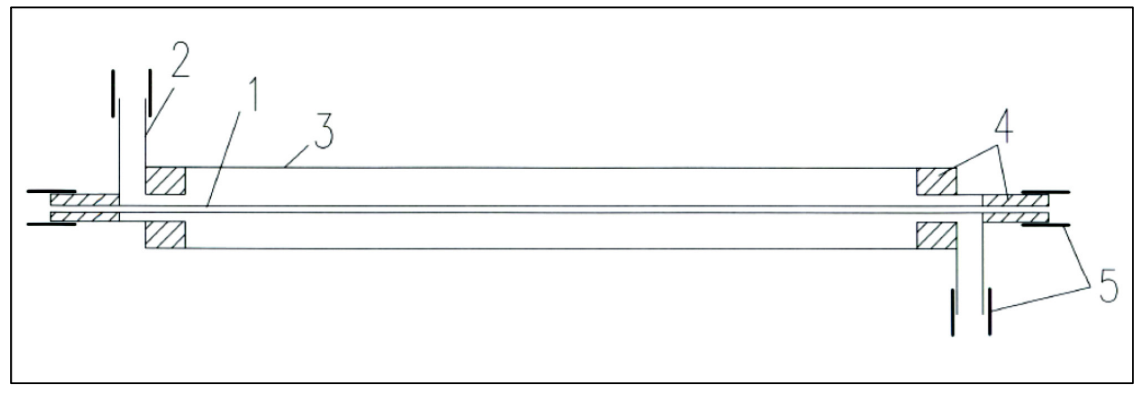

Figure 2: $\quad$ Scheme of a single-fibre module.

The counter-current mode of water and air flows was selected for this study on the basis of reference [1]. Modules with fibres were connected in a vertical position, when air flows from top to bottom. During experiments only one part of the equipment was operated (either a module with fibre bundle or a module with a single fibre). The Toluene concentration in the water in a storage tank was about $100 \mathrm{mg} / \mathrm{l}$.

Analyses of toluene in water and air after its capture on activated carbon were performed. Analyses of toluene in aqueous samples were done with gas chromatography with mass spectrometry by using headspace injection (Agilent Technologies 7890A/5975C inert, autosampler CTC CombiPal with a headspace unit). Analyses of toluene in the air were performed after the extraction of activated carbon adsorption tubes by carbon disulphide and consequent analyses by gas chromatography with flame ionisation detection (Agilent Technologies $6890 \mathrm{~N})$.

\subsection{Materials}

Membranes consisting of three various polymeric materials were tested during experiments. The materials were PE, PP, PVDF (Table 1).

\section{Influence of water flow on toluene separation efficiency}

The experiment was conducted with fibre-bundle membranes; for the reason that they are commercially available and they are commonly used for water purification. The bundle can be considered as a separation unit although bundles 
Table 1: $\quad$ Parameters of used materials.

\begin{tabular}{|c|c|c|c|}
\hline Materials & $\begin{array}{c}\text { Outer fibre } \\
\text { diameter } \\
(\mathrm{cm})\end{array}$ & $\begin{array}{c}\text { Specific fibre } \\
\text { surface } \\
\left(\mathrm{cm}^{2}\right)\end{array}$ & Fibre length \\
$(\mathrm{cm})$
\end{tabular}

from various manufacturers can be different in some parameters. The used method is based on the assumption that the toluene water concentration will decrease after passing through module with membranes.

Influence of water flow on separation efficiency was evaluated by using a bundle with a PP membrane containing approximately 1500 fibres (according to the manufacturer). The experiment was performed under flows of one, three and six $1 / \mathrm{min}$. The air flow was set so that toluene was effectively removed from fibres; in all cases it reached $14 \mathrm{l} / \mathrm{min}$. Separation efficiency was calculated from toluene concentration values in aqueous samples before and after the module. The values of separation efficiency for individual water flows are given in Table 2.

Table 2: Influence of water flow through the module on toluene separation efficiency.

\begin{tabular}{|c|c|c|c|}
\hline $\begin{array}{c}\text { Water flow } \\
(1 / \mathrm{min})\end{array}$ & $\begin{array}{c}\text { Toluene concentration } \\
\text { before module }(\mathrm{mg} / \mathrm{l})\end{array}$ & $\begin{array}{c}\text { Toluene } \\
\text { concentration after } \\
\text { module }(\mathrm{mg} / \mathrm{l})\end{array}$ & $\begin{array}{c}\text { Separation } \\
\text { efficiency }(\%)\end{array}$ \\
\hline 1 & 62 & 41 & 34 \\
\hline 3 & 56 & 43 & 23 \\
\hline 6 & 52 & 46 & 12 \\
\hline
\end{tabular}

The results showed that when the water flow increases the efficiency of toluene separation from water decreases. The efficiency was about $12 \%$ at the flow rate of $6 \mathrm{l} / \mathrm{min}$; but it was mostly three times higher at $11 / \mathrm{min}(34 \%)$.

\section{Hollow-fibre material testing}

Hollow-fibre membranes are produced from various materials [2-4]. Materials increasing separation efficiency are tested for various separation processes. We chose testing of material properties in single fibre modules in consequent experiments. Influence of events that could add some uncertainties to evaluation (adhesion between fibres) were eliminated in this way (Fig. 2). Changes in 
toluene concentration values in water are negligible after passing through the fibre, so that separation efficiency is calculated from the quantity of the removed toluene which passed through the membrane wall to the air flowing on the inside of the fibre. The air passed through an activated carbon adsorption tube that captured the separated toluene. The calculated values of removed toluene relative to an area unit in specific time are given in Table 3.

Table 3: Quantities of removed toluene relating to membrane materials.

\begin{tabular}{|c|c|}
\hline Material & $\begin{array}{c}\text { Removed toluene related to } \\
\text { surface unit }\left(\mathrm{ng} / \mathrm{cm}^{2}\right) \text { in 30 min }\end{array}$ \\
\hline PE & 208.7 \\
\hline PP & 179.0 \\
\hline PVDF & 017.4 \\
\hline
\end{tabular}

PE and PP membranes have very similar capabilities for separating toluene from water. It can be caused by the similar structure of these materials. PDVF fibre showed 10 times lower separation efficiency; it could be considered to be unsuitable for application in water purification processes.

\section{Conclusion}

The special laboratory equipment was built to observe the various parameters of hollow-fibre membranes used for toluene separation from water. The pilot experiments were conducted with commercially available membranes in this equipment.

This study is aimed at experiments observing two important characteristics which can significantly influence toluene separation efficiency from water. The first was the water flow rate through the fibre-bundle module, the second was the material of the used membranes.

It was found that the water flow rate through the fibre bundle module has an important influence on toluene separation efficiency from water. The fact is important with respect to the practical use of hollow-fibre membranes in water treatment.

Single-fibre modules were prepared for evaluation of individual membrane materials and it was verified that choice of membrane material could be essential for effective applications. In this study the polyethylene fibres were determined as the most suitable material for toluene removal from contaminated water. Further experiments are planned to develop presented observations.

\section{Acknowledgements}

Experiments described in this study were realised with financial support of the Technology Agency of the Czech Republic, project number TA 01010552 and in connection with project Energy Units for Utilisation of Non-Traditional Energy 
Sources, reg. no. CZ.1.05/2.1.00/03.0069, supported by Research and Development for Innovations Operational Programme financed by Structural Funds of the European Union and from the means of the state budget of the Czech Republic.

\section{References}

[1] Qin, Y.J., Cabral, J.M.S., Lumen mass transfer in hollow-fibre membrane processes with constant external resistances, AIChE Journal, 43(8), pp. 1975-1988, 1997

[2] Penga, M., Vane, L. M., Liu, S. X., Recent advances in VOCs removal from water by pervaporation, Journal of Hazardous Materials, B98, pp. 69-90, 2003

[3] Cai, B. X., Polymer membranes for separating organic mixtures, Journal of Applied Polymer Science, 101(2), pp. 1160-1164, 2006

[4] Yahaya, G.O., Separation of volatile organic compounds (BTEX) from aqueous solutions by a composite organophilic hollow fibre membranebased pervaporation process, Journal of Membrane Science, 319(1-2), pp. 82-90, 2008 